\title{
Assessment of the Clinical, Serological and Parasitological Aspects of Onchocercal Skin Disease, 20 Years of Community Directed Treatment with Ivermectin (CDTI) in the Ruvuma, Southwestern Tanzania
}

\author{
WH Makunde ${ }^{1 *}$, AR Mawson ${ }^{2}$, F Francis ${ }^{1}$, ZXN Savael ${ }^{1}$, A Kubeja ${ }^{4}$, G Komba ${ }^{3}$, R Ngowi $^{3}$, S \\ Rubinchik ${ }^{5}$ and Alan D Penman ${ }^{6}$ \\ ${ }^{1}$ National Institute for Medical Research Tanga Centre, United Republic of Tanzania, Tanzania \\ ${ }^{2}$ School of Health Sciences, State University, United States of America \\ ${ }^{3}$ Songea District Hospital, Songea, United Republic of Tanzania \\ ${ }^{4}$ National Institute for Medical Research Tukuyu Centre, United Republic of Tanzania,
}

${ }^{5}$ Department of Medicine, University of Mississippi Medical Centre, United States of America

${ }^{6}$ Department of Microbiology, University of Mississippi Medical Center, United States of America

*Corresponding author: WH Makunde, Research Fellow, National Institute for Medical Research Tanga Centre, Tanga, United Republic of Tanzania.

To Cite This Article: WH Makunde, AR Mawson, F Francis, ZXN Savael, A Kubeja, et al., Assessment of the Clinical, Serological and Parasitological Aspects of Onchocercal Skin Disease, 20 Years of Community Directed Treatment with Ivermectin (CDTI) in the Ruvuma, Southwestern Tanzania. Am J Biomed Sci \& Res. 2021 - 13(1). AJBSR.MS.ID.001836. DOI: 10.34297/AJBSR.2021.13.001836.

Received: 眥 May 25, 2021; Published: 眥 June 08, 2021

\begin{abstract}
Background: Onchocerciasis is a chronic disease caused by filarial worm, maintained in the communities through the black flies that breed in rapid fast flowing rivers. The disease triggers enormous sufferings and compromise community and individual socio-economic opportunities. Approximately 37 million people are afflicted by onchocerciasis and 46,000 new cases of blindness are reported each year in the hyper-mesoendemic communities. Study objective was assessing interruption of transmission after over 20 years of mass drug administration in the area.

Methods: In this descriptive study design, clinical signs, symptoms and skin snip results were the parameters used to diagnose Onchocercal skin disease (OSD) in hyper and hypo/non-endemic communities. Quantitative PCR was used to confirm the diagnosis and determine the number of onchocerca volvulus (o.v.) genomes present in the skin specimens of onchocerciasis cases and non-infected cases.

Results: A total of 106 individuals participated in this study, 59.4\% (63/106) were males. The median age was 49.5 years with an inter-quantile range of 36 to 62 years and the mean weight was $55.7 \mathrm{~kg}$ with a $95 \% \mathrm{CI}$ of 53.8-56.7 kg. Microfilaridermia prevalence in the hyper and hypo / non endemic communities was $48.1 \%(51 / 106)$ and $1.0 \%(2 / 228)$ respectively with geometric mean intensity GMI of $2.8 \mathrm{mff} / \mathrm{mg}$ skin with $95 \%$ CI of 2.5-3.1. The prevalence of troublesome itching was 9.6\% (49/513) the hyper and hypo/non endemic communities respectively. Chronic papular onchodermatitis (CPOD) prevalence in the hyper-endemic villages was $24.5 \%(26 / 106)$ presented by itching along with scratch marks. An association between CPOD and microfilaridermia was observed ( $r=-0.27 ; p=0.047)$ while in the hypo and non-endemic communities the most frequent skin lesion observed was scabies and a red scaly skin rash. The amounts of 0.V. DNA detected in the specimen varied greatly. Twelve participants had data on O.V. DNA and expressed higher levels of O.V. DNA (r2 = 0.25).
\end{abstract}

Conclusion: These findings have shown clearly that, transmission has not been interrupted in the entire focus. However, in areas considered non endemic in the previous surveys today transmission is ongoing.

Keywords: Onchocerciasis; Chronic Disease; Ivermectin; Microfilaridermia; Skin Itching; Songea; Tanzania 
Abbreviations: CDTI: Community Directed Treatment with Ivermectin; WHO: World Health Organization; MDA: Mass Drug Administration; DOT: Direct Observed Treatment; OSD: Onchocerca skin Disease; CML: Community Microfilarial Load; TNF $\alpha$ : Tumour Necrosis Factor; IL-L $\beta$ : Interleukin Beta; NO: Nitric Oxide; DNA: Deoxyribo Nuclei Acid; RNA: Ribonucleic Acid; rPCR: Real Time Polymerase Chain Reaction; APOD: Acute Popular Onchodermatitis; LOD: Lichenified Onchodermatitis; CPOD: Chronic Popular Onchodermatitis; DPM: Depigmentation; Mf: Microfilaria; O.V: Onchocerca Volvulus; BMI: Body Mass Index; SD: Standard Deviation; GAPDH: Glyceraldehyde 3-phosphate dehydroqese; S.E.M.: Standard Error of the Mean;IQR: Interquartile Range; TZNOCP: Tanzania National Onchocerciasis Control Programme

\section{Introduction}

Onchocerciasis is a parasitic disease caused by Onchocerca volvulus, a filarial nematode transmitted by black flies (Simulium $s p$.) The clinical expression of the disease is characterized by dermatologic and ocular manifestations namely; itching, pruritis, dermatitis, depigmentation, pre-matured skin atrophy, discoloration, and ocular lesions in the anterior and posterior segments of the eye. Over time, accumulated ocular damage can result in visual loss and blindness, the disease is ranked as the fourth leading infectious cause of preventable blindness [1]. It is estimated that, 37 million people are infected [2] with 46,000 annually new cases of blindness (http://www.apoc.bf/). The disease is endemic in sub-Saharan Africa and phylogenetdesowitzically proved to have relationship between African Old World and America New world (https://graduadeway.com/onchocerciasis-in-latin-America/. It is for this reason that, parasite onchocerca volvulus is known to be imported from the West Africa to Americas through the slave trade [3] and spread further through migration [3]. Although the disease was introduced from Africa, it was first described in 1915 by Rodolfo in Guatemala [4]. The ocular and dermatological morbidities together with the abandoned of individuals from the very fertile land in West Africa triggered the international organizations such as WHO to plan for control programme which was named Onchocerca control programme (OCP) in some 11 countries of West Africa. This was the area where the savannah strain of parasite causing blindness $[5,6]$ are residing. On the other hand, another strain, the forest type non blinding was responsible for the severe skin disease, commonly found in Central \& East Africa $[7,8]$.

The introduction of ivermectin a veterinary medicine into the management of filarial diseases in humans was a breakthrough during the 1987. This was also the time the drug was registered and licensed as a drug for treating onchocerciasis and lymphatic filariasis. Since then, control measures have been intensified in some endemic areas in the Americas and Sub-Saharan Africa. However, the use of ivermectin as a control tool has had an impact on the disease prevalence leading to increased reports of an increasing successful story on interrupting transmission in the endemic foci of the Americas, West and East Africa [9-13]. On the other hand, this recent successful control activity was attributed to annual/semi-annual rigorous and sustained community directed treatment with ivermectin (CDTI). Since ivermectin is only lethal to the larval stage (microfilariae) but not the adult parasite, prolonged treatment rounds at semi-annual might halt transmission in those endemic areas mostly the hotspots. Moreover, the proportion of animal-filariae in the vector has direct and indirect consequences for parasite transmission to humans $[14,15]$ causing an important factor to understand the epidemiology of the disease. Additionally, filariae closely-related to nematode onchorca volvulus might repopulate the human host [16] contributing to a potential risk of infection or could transfer genes to 0 . volvulus which could negatively alter effectiveness of ivermectin [17,18]. After the closure of the OCP control programme, which were targeting the simulium vectors using insecticides, a need to proliferate continuation of the control activities was crucial hence adopting a new strategy the chemotherapy [19-22]. Around 1987 a veterinary drug ivermectin was licensed to treat human helminthic disease and therefore in 1995 an alternative programme was formed, the African Programme for Onchocerciasis Control (APOC), to take up the control activities using chemotherapy this time, the ivermectin which was supported by Mectzan as donation for the entire period as required until the disease is eliminated in 20 countries including Tanzania. In Tanzania, prevalence rates of onchocerciasis in endemic areas range widely, from $3.5 \%$ to $63.6 \%$ in the known foci $[23,24]$. The epidemiology of the disease has also changed in recent years, possibly due to local environmental and global changes in climate and human populations as well as parasite and vector dynamics and heterogeneity in the human host response [25]. It could also reflect natural attrition of mff, which secrete Wolbachia bacterial endosymbiont products into the general circulation, inducing immune responses and inflammatory cytokines, resulting into pathologies in different organs $[26,27]$ including the brain possibly provoking the different forms of epileptic seizures due to leiomodin 1 in a repeatedly manner $[28,29]$. In endemic areas, $40-50 \%$ of adults are symptomatic, with devastating socioeconomic consequences whereby entire villages are abandoned. The effectiveness of the sole treatment, ivermectin, is declining and there is increasing resistance to this drug [30-32]. There is therefore an urgent need to understand the pathogenesis and current epidemiological status of the disease in those endemic foci as a basis for developing new control and treatment strategies towards elimination of onchocerciasis. 
In Tanzania, onchocerciasis is prevalent in 15 districts which lie within the tropical rain forest. In these areas, the epidemiological profile has been experiencing environmental changes in favor of the parasite and vector creating an increased threat to the human population in the area. According to previous endemicity data in those same foci it appears that, transmission is still on ongoing. Those foci are linked by the Eastern Arc Mountain chain of Block Mountains which stretches from northeast Tanzania to Northern Malawi and from the Tanga region to the Ruvuma and south-western Mbeya regions in Tanzania [33,34]. In 1993 WHO estimated 650,000 individuals were infected with onchocerciasis in Tanzania but there is a wide variation in the disease prevalence in the different disease foci $[35,25,36,23]$.

It has been difficult to control Onchocerciasis by targeting the simulium vectors because the breeding ecology is confined to thick forest and fast flowing rivers in the arc of Block Mountains in eastern Tanzania. These breeding sites are not always accessible by land, although through aerial spraying these regions could be accessed, but resources are limited to enter into such control ventures. In Tanzania the introduction of Community Directed Treatment with Ivermectin (CDTI) through APOC in 1997, it has represented the best strategy for onchocerciasis control in Tanzania. This strategy was initially implemented at the Mahenge focus, subsequently extended to Ruvuma (1998), Tanga and Tukuyu (1999), Kilosa (2003), Morogoro (2004) and finally the Tunduru focus (2005) $[37,38]$. It is unfortunate that before the control programme was introduced in the country pre-treatment baseline data for monitoring and evaluation of the programme or ivermectin impact after the desired period of mass treatment was overlooked. The current study measures the status of clinical disease and infection using parasitological, serological and molecular tools (PCR) in order to assess the impact of CDTI in four districts (Namtumbo, Mbinga, Songea rural (Madaba) \& Urban) in the Ruvuma region of southern-western Tanzania.

\section{Materials and Methods}

\section{Study Area}

Songea is the largest single onchocerciasis focus within Ruvuma region in the southwestern Tanzania. It is composed of Songea which lies between latitudes $10.64 \mathrm{~S}$ and longitudes $35.64 \mathrm{E}$, Mbinga situated between latitudes 10.94 S and longitudes $35.00 \mathrm{E}$, and Namtumbo which lies between latitudes $10.47 \mathrm{~S}$ and longitude 36.13 E. This focus borders Mozambique to the south and Lake Nyasa to the west. The three districts; Mbinga, Songea and Tunduru were and are endemic to date. The focus covers around 15,000 $\mathrm{km}^{2}$ and is characterized by a number of river systems that flow from and traverse the Livingstone mountain range into Lake Nyasa. The Mbinga district is characterized by five river systems, the Ruvuma, Ruhuhu, Mnyamaji, Mngaka and Lumeme, which provide ideal simulium vector breeding sites and maintain onchocerciasis transmission within the focus.

Study Design and Sampling Process : A descriptive crosssectional observational study carried out in four districts of the Ruvuma region in the southwestern Tanzania. Study communities were selected according to their endemicity levels/status, being hyper-endemic, meso-endemic, hypo-endemic or non-endemic, on the basis of endemicity levels from historical data $[23,36]$. Study investigators provided an explanation and the project aims to district authorities and community leaders thereafter meetings with community health leaders and administrators were conducted to sensitize the study population. The discussion focused on the current status of OSD after ivermectin distribution in those areas. After such meetings, permission to carry out the study at each local setting was granted. Thereafter, local leaders asked community members willing to participate in the study to visit the local health facility (village dispensary/health centre) where written and oral informed consent was obtained. The next day those who consented came back for a physical examination and a blood specimen and skin snips were collected together with dietary intake information.

Physical Examination: Clinical diagnosis of onchocerca skin disease was conducted by a physician experienced in onchocerciasis using [39] criteria for skin grading. An interview on the occurrence of itching preceded the physical examination. In the latter, each individual's skin was examined, beginning with the head, then followed by the neck, upper limbs, anterior and posterior chest, abdomen, genitals, back, buttocks and lower limbs. Following this clinical examination, any skin lesions detected were recorded according to [39]. Individuals who presented other dermatological lesions not compatible with OSD were also recorded.

Visual acuity of each individual was also investigated by an ophthalmologist using Snellen's letter chart for those who could read or Sjogren's hand test for those who were illiterate, at a distance of 6 meters from the individual. Similarly, the anterior chamber and fundus of each eye were examined, using a slit lamp directly or indirectly by using an ophthalmoscope. Both eyes of each individual were checked for fluffy opacities in the cornea, sclerosing keratitis, and the presence of microfilariae (mff), after an individual sat down inclined for two minutes.

Parasitological Examination: Skin snipping using corneoscleral biopsy punch was performed on each study participant taking skin snips from the right and left iliac crest, pelvic girdle, right and left buttocks. Before snipping was conducted, the skin was disinfected with $70 \%$ alcohol, and allowed to dry prior to snipping. The skin snips were immersed in $200 \mu \mathrm{L}$ of $0.9 \%$ normal saline in 96 well microtiter plates. Parafilm was used to seal the plates and the plates were wrapped in a piece of damp tissue in a petri dish to prevent drying of the specimens. The specimens 
were transferred immediately to the laboratory for microscopy to quantify mff. All samples were labeled in coded fashion to conceal the identity, diagnosis and case/control status of the patient. Each snipped skin specimen was then blotted to remove excess moisture and weighed on an electronic balance to allow expression of the intensities of infection as mff/mg skin snip.

DNA and RNA Extraction and Quality Assessment: Total genomic DNA and total RNA were extracted from each skin specimen using a Fisher BioReagents SurePrep RNA/DNA/Protein Purification Kit and evaluated using quantitative PCR (qPCR) with appropriate negative controls. Quality and quantity of genomic DNA recovered from the samples was evaluated with NanoDrop spectroscopy. One in-house blood specimen (not associated with this study) was also processed with this kit in order to obtain a negative control of human DNA and RNA. A custom TaqMan assay for detection of $O$. volvulus genomic DNA (Ovol2) was designed and synthesized at Applied Biosystems. Although the results of individual samples varied significantly, all specimens were submitted for qPCR analysis.

Similarly, RNA samples were also analyzed with NanoDrop spectroscopy and most appeared to have a sufficient quantity of RNA for testing. A set of 8 RNA specimens were evaluated with an Agilent 2100 Bioanalyzer (Agilent Technologies) for RNA quality/ integrity. While the assay control (extracted from whole blood) had good quality RNA with a RIN (RNA integrity number) value of 9.3, the skin snip specimens had highly degraded RNA, with very low RIN values. Nevertheless, all RNA samples were used to prepare cDNA with the SuperScript III First Strand Synthesis System (Thermo Fisher Scientific, Inc.), using 7.5ng RNA per reaction (based on NanoDrop readings).
DNA and RNA Analysis: Genomic DNA specimens were evaluated with the 0vol2 assay for the presence of 0 . volvulus genomes. Relative parasite copy numbers were normalized to the copy number of human genomic DNA as determined by a GAPDH (glyceraldehyde 3-phosphate dehydrogenase) assay and reported as fold averages \pm S.E.M. relative to the levels observed in the negative assay control. Average Ct values obtained for GAPDH assay indicated that the total amount of human DNA detected by the assay was similar in most samples with some exceptions (typically related to very low DNA quantity and/or quality).

\section{Results}

Eleven communities in four different districts of the Ruvuma region were examined in order to identify suitable individuals for the study (Table 1); 176 individuals from four villages in Mbinga district, with $1.1 \%(2 / 176)$ mff positive, 166 individuals from two villages in Namtumbo district with $12.0 \%$ (20/166) mff positive, whereas the prevalence of microfilaridermia in 347 individuals from four villages in the Songea rural district was 8.9\% (31/347) and 52 individuals in a single village in the Songea urban district were all mff negative. The total number of individuals examined during the cross-sectional survey was (741), $54.4 \%$ (403/741) were males. Those individuals fulfilling inclusion criteria were 106, aged 18 years and above, hence interviewed for the study. Of which 59.4\% (63/106) were males, majority, 52.0\% (55/106) were from non-endemic village (Figure 1). The mean weight of study participants was $55.7 \mathrm{~kg}$ with a $95 \%$ CI of $53.8-56.7 \mathrm{~kg}$ and the median age was 50 years old with an interquartile range (IQR) of 36-60 years old (Table 2). A high proportion (67.6\%) of the study participants had acquired primary or secondary education, while $32 \%$ did not attend any formal school and the primary occupation of over $90 \%$ of the participants were peasant.

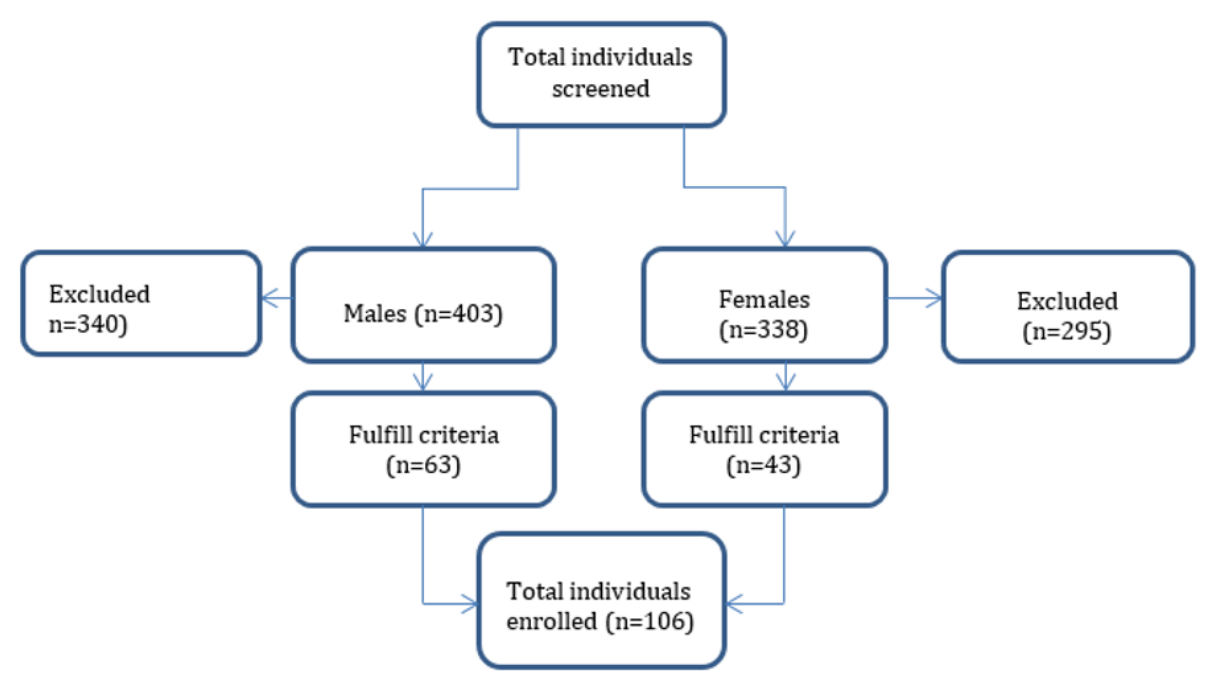

Figure 1: Study Profile During Recruitment of Participants Process in Ruvuma Tanzania. 
Table 1: Screening and enrollment of study participants by district and community in the Ruvuma Region, Tanzania.

\begin{tabular}{|c|c|c|c|}
\hline District & Village & Screen, $\mathbf{n}(\%)$ & Mf positive , $\mathbf{n}(\%)$ \\
\hline \multirow{4}{*}{ Mbinga (n=176) } & Mtua & $68(38.6)$ & $2(2.9)$ \\
\cline { 2 - 4 } & Mipotopoto & $41(23.3)$ & $0(0)$ \\
\cline { 2 - 4 } & Luhanga & $2(1.1)$ & $0(0)$ \\
\cline { 2 - 4 } & Masimeli & $65(36.9)$ & $0(0)$ \\
\hline \multirow{3}{*}{ Namtumbo (n=166) } & Mkongo & $66(39.8)$ & $0(0)$ \\
\cline { 2 - 4 } & Kitanda & $100(60.2)$ & $20(20.0)$ \\
\cline { 2 - 4 } Songea Rural \& urban (n=399) & Mtanygimbole & $151(43.5)$ & $24(15.9)$ \\
\cline { 2 - 4 } & Matimira & $76(21.9)$ & $2(2.6)$ \\
\cline { 2 - 4 } & Liwete & $67(19.3)$ & $5(7.5)$ \\
\cline { 2 - 4 } & Liganga & $53(15.3)$ & $0(0)$ \\
\hline
\end{tabular}

Table 2: Demographic characteristics of the 106 study participants from the Ruvuma region.

\begin{tabular}{|c|c|c|c|}
\hline Variable & Categories & $\mathbf{N}$ & $\mathbf{\%}$ \\
\hline \multirow{2}{*}{ Sex } & Male & 63 & 59.4 \\
\hline Age & Female & 43 & 40.6 \\
\cline { 2 - 4 } & Median (IOR) & 50 & $30-60$ \\
\hline Weight in Kg & Mean (95\% CI) & 55.7 & $53.4-56.7$ \\
\hline Body max index & Mean (range) & 23 & $15.6-33.7$ \\
\hline Education level & None & 34 & 32.4 \\
\hline \multirow{3}{*}{ Occupation } & Primary & 63 & 59.1 \\
\cline { 2 - 4 } & Secondary & 9 & 8.5 \\
\cline { 2 - 4 } & Peasant & 96.6 \\
\cline { 2 - 4 } & Student & 2 & 1.9 \\
\cline { 2 - 4 } & Employed & 7.5 \\
\hline
\end{tabular}

$I Q R$, interquartile range; $\mathrm{Cl}$, confidence interval

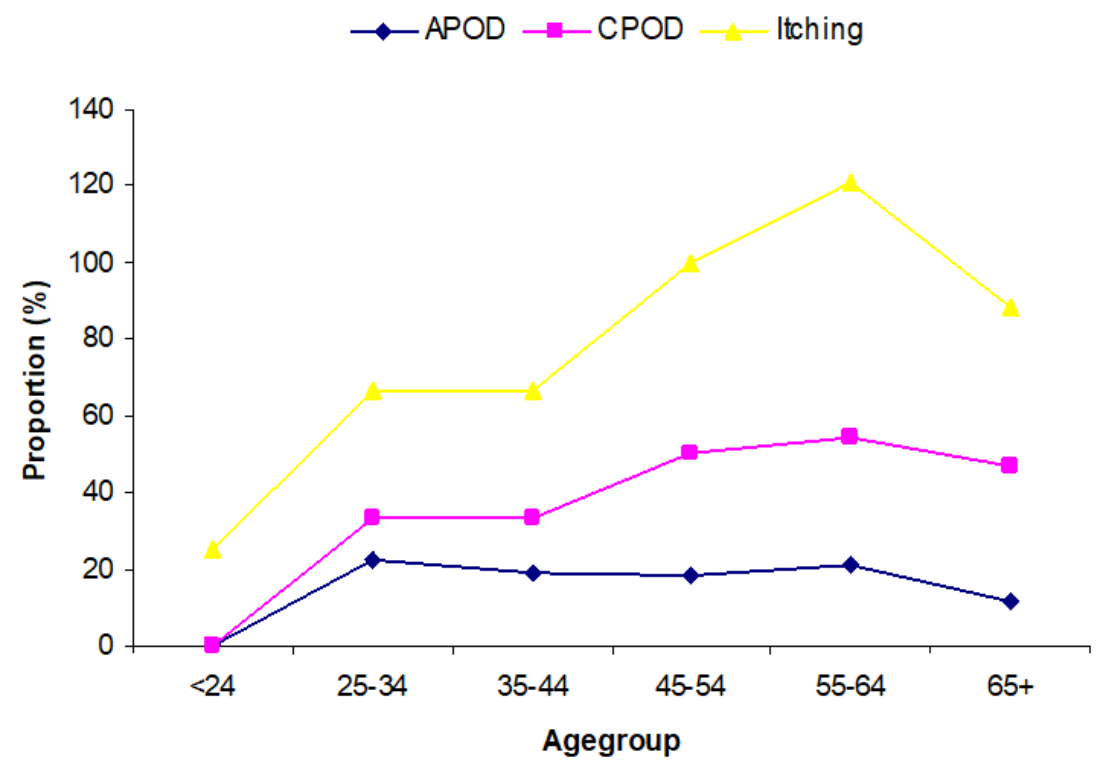

Figure 2: Illustrates the Relatiosnship between Age and Presence of Clinical Features; APOD, CPOD \& Itching. APOD=Acute Popular Onchodermnatitis, $C P O D=$ Chronic Popular Onchodermatitis 
(Figure 2) illustrates the relationship between age and the presence of clinical diseases features. Chronic papular onchodermatitis (CPOD), acute papular Onchodermatitis, (APOD) and itching were the common clinical features observed among studied participants. The chronic onchocercal pathologies begin after the adolescent age and progress with age, reaching a peak between 55 and 64 years then finally a slight decrease in the most elderly age.

(Figure 3) Illustrates the proportion of clinical disease and $\mathrm{mf}$ during the three years follow up. The mf proportion when compared with the baseline and the subsequent follow up 2017 and 2018, there was a reduction from $48 \%$ to $<20 \%$ and $12 \%$. whereas APOD at baseline was $17 \%$ and the 2017 follow up was $<1 \%$, however, in 2018 the APOD rates increased to 19\%. On the other hand, atrophy was increasing, at baseline (2012) was 15\% whereas 2017 increased to $17 \%$ and (2018) at 28\%. CPOD at baseline (2012) was $22 \%$ and the second follow up (2017) increased to almost double $40 \%$ although in the last follow up, there was a large proportion of $32 \%$ reduction in CPOD conditions. The proportion of DPM was maintained only a big change was observed during the last follow up 2018 from $12 \%$ to $8 \%$, there was a change in proportion of people presenting itching as compared to baseline 2012 (46\%) and the last follow up 2018 (12\%) (Figure 3).

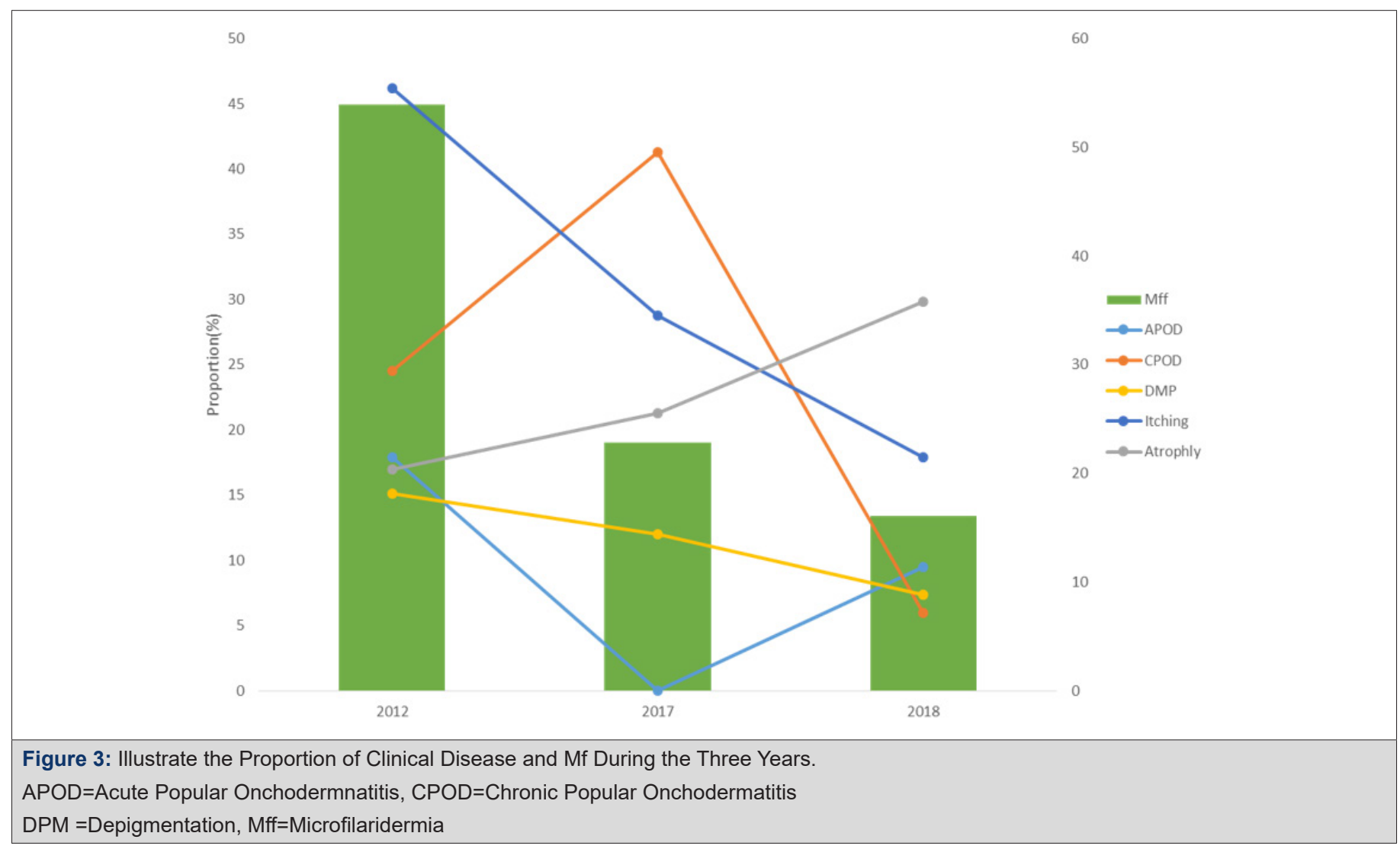

Onchocercal skin lesions and microfilaradermia observed in the study population

The vast majority of these onchocercal skin lesions were observed in study participants from endemic villages; $49 \%$ with CPOD, 35.3\% with APOD or Atrophy, 27.5\% with DPM, and $90.2 \%$ suffered from dermal itching (Table 3). Only a single individual exhibited lichenified onchodermatitis. The occurrence of chronic onchocercal skin lesions and itching was nearly absent in the hypoendemic/non-endemic villages with only 2 individuals, both from the Matimira village, afflicted with the chronic skin lesions. All individuals presented with one of the chronic dermal pathologies of onchocerciasis (APOD, CPOD, atrophy, DPM, or itching) were positive for microfilaria in their skin snip specimen. Of these, 51 were from endemic villages and 2 were from the non-endemic (Matimira village). Table 4 shows the anatomical location where mff-positivity were detected, of those 19 and 26 presented APOD and CPOD respectively. APOD was the common skin lesion observed on the arms, abdomen, shoulder, back, buttocks and legs. However, lesions on the head, neck, chest, groin, and perineum were much less common, observed in less than $15.8 \%$ of individuals. Those individuals with COPD, were commonly observed on the arms, abdomen, back, buttocks and legs with proportions ranging from $73.1 \%$ to $100 \%$. Whereas lesions on the head, neck, chest, shoulder, groin, and perineum were less common, each observed in less than $38.5 \%$ of the individuals. 
Table 3: Prevalence of skin lesion among recruited study participants.

\begin{tabular}{|c|c|c|c|c|}
\hline & \multicolumn{4}{|c|}{ Year } \\
\hline Type of skin lesion & Base line (n=106) & $\mathbf{2 0 1 7}(\mathbf{n = 8 0}$ & $\mathbf{2 0 1 8}(\mathbf{n = 6 7 )}$ & P-value \\
\hline APOD & $19(17.9)$ & $0(0)$ & $2(9.5)$ & $\mathrm{p}<0.001$ \\
\hline CPOD & $26(24.5)$ & $33(41.3)$ & $4(6.0)$ & $\mathrm{p}<0.001$ \\
\hline Atrophy & $18(17.0)$ & $17(21.3)$ & $20(29.8)$ & 0.134 \\
\hline DMP & $16(15.1)$ & $10(12.0)$ & $5(7.4)$ & 0.328 \\
\hline Itching & $49(46.2)$ & $23(28.8)$ & $12(17.9)$ & $\mathrm{p}<0.001$ \\
\hline Mff+ve & $53(60.9)$ & $20(22.2)$ & $14(16.1)$ & $\mathrm{p}<0.001$ \\
\hline
\end{tabular}

Table 4: Proportion of troublesome itching and Microfilareidermia according to study villages.

\begin{tabular}{|c|c|c|c|}
\hline Study Villages & Mff+ve n (\%) & Troublesome itching n (\%) & Mff+ve and itching* \\
\hline Kitanda (n=100) & $20(20.0)$ & $19(19.0)$ & $19(95.0)$ \\
\hline Liweta (n=67) & $5(7.5)$ & $3(4.5)$ & $3(60.0)$ \\
\hline Mtua (n=68) & $2(2.9)$ & $2(2.9)$ & $1(50.0)$ \\
\hline Mtyangimbole (n=151) & $24(15.9)$ & $23(15.2)$ & $23(95.3)$ \\
\hline Lilambo (n=53) & $0(0.0)$ & $0(0.0)$ & $0(0)$ \\
\hline Matimira (n=77) & $2(2.6)$ & $2(2.6)$ & $0(0)$ \\
\hline
\end{tabular}

The mean age of APOD in the endemic villages was lower as compared to CPOD, Atrophy and DPM; on the other hand, the prevalence of those conditions was also high in the endemic villages than in the hypo-endemic/non-endemic villages. However, those individuals complaining of itching did also present onchodermatitis.

The mean mff total count (summed over 6 body sites) was 26.6 (median 15, min.1.0, max. 191.0), with a highly left-skewed distribution (data not shown). The mean itch score was higher among the individuals from endemic communities than among the individuals from the non-endemic/hypo-endemic communities (max. possible: 13) was 5.9 (min.0, max. 10). However, there was no correlation between mean mff total count and mean itch score $(\mathrm{r}=0.08)$.

Itching was observed as scratch mark in the following body regions namely; head and neck 1.9\% ( 2/106), anterior chest $8.5 \%$

Table 5: Community Microfiridermia load and OV $16 \lg 4$ according to individuals examined.

\begin{tabular}{|c|c|c|c|c|c|c|}
\hline \multirow{3}{*}{ Village } & \multicolumn{6}{|c|}{ Year } \\
\hline & \multicolumn{2}{|l|}{2012} & \multicolumn{2}{|l|}{2017} & \multicolumn{2}{|c|}{2018} \\
\hline & $\begin{array}{c}\text { CML; mean(range) mff/ } \\
\text { mg skin }\end{array}$ & OV 16 & $\begin{array}{l}\text { CML; mean(range) mff/ } \\
\text { mg skin }\end{array}$ & OV 16 & $\begin{array}{l}\text { CML; mean(range) } \\
\text { mff/mg skin }\end{array}$ & OV 16 \\
\hline Kitanda (58) & $23(16-33)$ & $\mathrm{Na}$ & $8(4-17)$ & $10 / 21(47.6)$ & $4(2-8)$ & $6 / 16(37.5)$ \\
\hline Liganga (53) & $2(1-3)$ & $\mathrm{Na}$ & 0 & $20 / 34(58.8)$ & 0 & $14 / 19(73.7)$ \\
\hline Liweta (13) & $10(3-41)$ & $\mathrm{Na}$ & 0 & $0(0)$ & $3(1-9)$ & $0(0)$ \\
\hline Mtyangimbole (53) & $12(7-20)$ & $\mathrm{Na}$ & $8(2-39)$ & $4 / 16(25.0)$ & $9(1-134$ & $5 / 13(38.5)$ \\
\hline Lilambo (80) & 0 & $\mathrm{Na}$ & 0 & $25 / 26(96.2)$ & 0 & $20 / 20(100)$ \\
\hline Matimira(47) & $24(1-44)$ & $\mathrm{Na}$ & $9(4-18)$ & $9 / 12(75)$ & 0 & $11 / 14(78.6)$ \\
\hline
\end{tabular}


Genomic Data in the Endemic and Hypo-/Non-Endemic Communities: The total genomic studied patient was 53, of those 25 expressed O.V. DNA, whereas 14 of those had raised O.V.DNA levels, with a very wide range, 3.3-fold -15,102-fold; 11 had normal /low O.V. DNA levels. Of the 53 controls, 22 had raised O.V. DNA levels with range 1.05-fold-393.4-fold; 2 had normal /low O.V. DNA levels Mawson et al. [36] Association between O.V. DNA levels and skin microfilaridermia count.

PCR results show that, DNA Genomic on the expression of O.v. DNA were available for 49 (45\%) of 106 participants. This group comprised 30 men and 19 women, with mean age 49.1 years (SD 13.3, min. 25, max. 65), mean BMI $23.8 \mathrm{~kg} / \mathrm{m} 2$ (SD 4.1, min. 16.0, max. 33.7). The amounts of O.v. DNA detected in the specimen varied greatly: 36 had raised $O . v$. DNA levels, with a very wide range (1.05-fold - 15,102-fold); 13 had normal/low O.v. DNA levels. The mean mff total count was 30.9 in the group of 14 with raised O.V. DNA levels, compared to 49.4 in the group of 11 with normal / low O.V. DNA levels. These results have shown that, there wasn't association between sex and microfilaria at the different villages involved in the study $(\mathrm{p}>0.05)$.

Atrophy and DPM: Skin atrophy was observed in four different body regions of the 18 affected individuals and this includes; both right and left thigh $88.99 \%$ (16/18), followed by back $83.8 \%$ $(15 / 18)$ and finally buttocks $88.9 \%(16 / 18)$. Majority of those were adult females $55.6 \%$ (10/18) while $44.4 \%$ (8/18) were males. Although DPM is among the frequent chronic OSD lesion observed in endemic villages in the rain forest type of disease, only few $15.1 \%$ $(16 / 106)$ were elicited with these conditions (atrophy and DPM) at the right and left anterior tibial region.

Other Skin Lesions Observed with Association with Onchocercal Skin Disease: Individuals with impaired visual acute according to the Snellen test were $4.7 \%$ (5/106) these were also mff positive individuals.

Palpable Nodules: Palpable onchocerca nodules were observed in $5.0 \%(5 / 106)$ of the study participants. Four were males and one female. Nodules were localized at the inguinal area while 3 were observed at the femoral area.

\section{Discussion}

\section{Clinical Disease, cellular and parasitological}

To assess whether treatments using CDTI and other ivermectin distribution strategies had effect on onchoceca volvulus in the endemic villages during the 20 years and beyond. The aim was to compare two similar setting with historical background data on transmission levels (meso-endemicity Vs hypo/non-endemicity) those studies, [23,36]. Therefore, clinical and parasitological methods were used to stratify the study participants into cases (mff+ve/dermatitis e.g. APOD, Scratch mark, LOD, CPOD DPM, Nodules \& Atrophy) and control (those did not present infections (mf), \& clinical disease (APOD, Scratch mark, LOD, CPOD DPM, Nodules \& Atrophy). Initially the study design was a case control, but during assaying and analysis, it was found that, about $92 \%$ of the participants drawn from hypo/non-endemic communities were positive also for Onchocerca volvulus genomic DNA and RNA by $\mathrm{rPCR}$, this then induced us to pool the data and carry out the analysis as a single group [36]. Clinically and parasitologically, one could suspect dealing with an area where transmission has been interrupted although this cannot substantiate through entomological and serological evidence to convince the observed phenomena. However, the clinical disease (dermatitis) and parasitological parameters alone do not meet the WHO criteria that transmission has been interrupted, [40]. Moreover, the study has shown, that, the prevalence and intensity of infection at Kitanda and Mtyangiambole was slightly higher than the hypo/non-endemic communities within the same geographical setting. Similarly, the prevalence of troublesome itching was also high in the two communities' considered hypo-non/endemic; although majority of the examined individuals were mff negative by microscopy. It was among these communities were mff by microscopy was "negative" however, through rPCR, twenty two out of twenty-four individuals considered negative had elevated genomic levels of $O$. volvulus DNA, indicating that, they were infected with $O$. volvulus and unsuspected sub-clinical $O$. volvulus infection despite having nether visible $\mathrm{mff}$ under the microscope and clinical sign of the disease. Hence the data from cases and control (non-cases) were pooled together and analyzed as one sample because a good number from the control were mff positive by rPCR although microscopically were mff negative. This is a sign of continuous low-level transmission in both endemic and hypo-non endemic communities, despite the 14 years of CDTI \& other ivermectin distribution treatments strategies in the study area. Possibly, this could have resulted from

1. The initial infections levels pre-treatments were high in the two settings than historical data from research background

2. Could also be the treatment coverage in the area was low than the WHO recommended levels [40]

3. It is also assumed that, drug distributors were not motivated through incentive package

4. Possibly advocacy was not sufficient to impact knowledge on the villagers the importance of taking the drug each round,

5. The other speculation, may be frequent multiple exposures of the drug, could have triggered tolerance or resistance of the nematode Onchocerca volvulus as observed in Ghana [31,32].

On the other hand, at Kitanda and Mtanygimbole the levels of infection (O. volvulus.) was higher as compared to the other villages 
raises the concern that, the parasite populations particularly their maturity (age) and the fecundity SlwSlow vnomic DNA levels indicating that they had micro-sub clinical $O$. volvulus infection. On the other hand, they did not present dermal mff or visible clinical signs or symptoms a feature of low transmission in the area. This was also the reason why study design was changed during the analysis [41-45].

\section{Itching \& Troublesome Itching}

Itching and pruritis was a frequent complaint presented in both endemic and hypo-non-endemic villages, though less pronounced in the non-onchocercal areas. On the other hand, the early clinical sign in OSD, is itching which present during immunological interaction between the parasite, human host and parasite products [TNF- $\alpha$, IL-Iß, NO, chemtaxin receptor \& netrophil chemotaxis [46] plus the Wolbachia endosymbionts even before pathological changes are observed. In the endemic communities it's not a surprise to see individuals using stones, wooden applicators and knives to scratch their skin because of itching to get relief of the symptoms. Surprisingly in the hypo/non-endemic villages clinical disease (oncho-dermatitis) was not a common feature, despite itching and scratching was a frequent reported feature in those communities. Although itching and reactive dermal lesions were common in all studied age groups in the endemic villages, they were generally present among the 20-40 age group which is the economically productive populations (Figure 2) involved in coffee and maize farming. This is an important aspect during the process of MDA since these peasants moved from their households to farms during the farming period and remained there until harvest period hence missing treatment. Therefore, extra effort is crucial to make sure such peasants are also covered during treatment with ivermectin through the CDTI strategy. In these endemic areas, the disease has features of rain forest, parasite strain whereby excruciating itching has been the main cause of morbidity [47] whereas visual impairment is uncommon. Elsewhere, such symptoms have been conflicting particularly adolescent females and women affecting marriage prospects [48], education, self-esteem [49], psycho-social alterations and neurological seizures [50,51]. Similarly reduced working capability and performance hence affecting individual socio-economic aspects [52]. Additionally, OSD-itching has been shown to significantly shorten the period of women to breast feed their neonates because of fatigue secondary to incessant itching [48].

\section{Why Transmission has Not been Interrupted in the Studied Area}

Why transmission has not been interrupted in this focus despite mass drug administration (MDA) has been implemented for over 15 years which is a long-term treatment using annual single drug strategy according to Tekle et al. [12] 2012) elsewhere [12]. Some possible factors which might contribute to this are discussed

1. The initial level of OSD prevalence and CML, could have been higher than what was observed on the ground

2. The type simulium damnosum s.l (cytoforms) which differs in distribution, ecology, behavior and ability to transmit the nematode worm onchocerca volvulus, therefore, a proper identification is crucial in order to understand the epidemiology of the disease in this particular area

3. individual compliance to treatments

4. coverage \& sensitization-advocacy could have been lower than expected

5. May be ivermectin distributors were not sensitized with attractive incentives as was done in Guinea [58] , training on the importance of completing the desired ivermectin rounds to those targeted population, administration of the drug adopting DOT strategy

6. May be those individuals failing to respond to ivermectin treatment, possibly there is an emerging drug resistance as has been reported in some areas in Ghana [31,32].

Indeed, geographical \& therapeutic coverage and compliance are among the most important factors that could have affected CDTI programme endpoints/outcome which generally dependent on social mobilization, supervision and monitoring as well as the adequacy of resources, not to forget the political willingness and commitment. In this study, we are referring to the entire focus because, this was the first epidemiological study carried in the area since ivermectin was introduced as CDTI/MDA. However, some control activities were initiated in this focus through religious faithoriented groups. Therefore, this study could particularly be valuable because it has shown that, despite the existence of CDTI/MDA over 15 years, transmission has not been interrupted within the human population in various studied villages in the focus probably there some hotspots fueling the transmission. On the other hand, there were some limitations while executing the study, some of those includes;

1. The design which was initially planned as case control but at the point of analysis we found that even in the areas considered non-endemic according to previous historical data, [23-25] a high infection rates was observed through PCR.

2. Some individuals were not able to be examined because, the mountainous topography of the area, hindered some of the said population be reached by motor vehicle or even motorcycle during the survey.

The results of this work indeed have highlighted the need to reassessing the programme in terms of treatment coverage level and comply in accordance to WHO recommendations, effective advocacy 
and health education to the ultimate targeted populations. Similarly carry out evaluation of the treatment activities, entomological, parasitological and serological in areas considered as hotspots where transmission interruption has not been achieved within the focus.

\section{Conclusion/Interpretation}

These results have shown clearly that within the focus, transmission has not been interrupted in the entire focus although exists signs of some villages having low infection and others appears that infection has stopped. However, in areas considered non endemic in the previous surveys today transmission is ongoing.

\section{Acknowledgements}

The author would like to thank all technical staff of Songea rural, Mbinga, Madaba and Namtumbo districts hospitals and the directors of the Songea rural, Songea urban, Madaba and Namtumbo councils. We would also like to thank the Director General of the National Institute for Medical Research, Professor Yunus Mgaya, the study participants who made this study possible; the Tanga Centre staff who assisted in the field (Mr Charles Guzo, salum Tembo, Thomas Semdoe and the Current Director of Tanga Medical Research Centre Professor John A P Lusingu for their good working environment and coordination of the project; and Lydia Lugomoro, the Centre accountant, for managing the project financial reports and statements.

\section{Conflict of Interest}

The Author (s) declared no potential conflicts of interest with respect to this work, authorship, and or publication of this manuscript.

\section{References}

1. WHO (2019) Blindness and vision impairmsent fact sheets. Geneva: World health Organization

2. Apoc 2005 (2005). African programme for onchocerciasis control launched 1995 as unique global partnership APOC 1995.

3. Desowitz RS (1981), discussed the history of the onchocerciasis, causes, and possible treatment of river blindness in his book. (Tales of Parasites and People)

4. Robles Robles (1915) was a first Guatemalan physician who correctly described the onchocerca volvulus in Latin America.

5. Anderson J, Fuglsang H, Hamilton PJS, Marshall TF De C (1974). Studies on Onchocerciasis in the United Republic of Cameroon. II. Comparison of Onchocerciasis in rain forest and Sudan savannah. Transaction of the Royal Society.

6. Peter H Adler, Robert A Cheke, Rory J Post (2010) Evolution, epidemiology and, population genetics of black flies (Diptera: Simuliidae). Infect Genet Evol (7): 846-865.

7. A Kalinga , R J Post (2011) An apparent halt to the decline of simulium woodi in the Usambara foci of onchocerciasis in Tanzania Ann.Trop. Ann Trop Med Parasitol 105(3): 273-276.
8. Adam Hendy, Andreas Kruger, Kenneth Pfarr, Jacobus De Witte , Addow Kibweja, et al. (2018) The blackfly vectors and transmission of Onchocerca volvulus in Mahenge, south eastern Tanzania. Acta Trop 181: 50-59.

9. Rodrigo J Gonzalez, Nancy Cruz Ortiz, Nidia Rizzo, Jane Richards, Guillermo Zea Flores, et al. (2009) Successful interruption of transmission of onchocerca volvulus in the Escuintla-Guatemala focus, Guatemala. PloS Negl Trop Dis 3(3): 404.

10. Rodriguez Perez MA, Unnasch TR, Dominguez Vazquez A, Morales Castro AL, Pena Flores GP, et al. (2010) Interruption of transmission of onchocerca volvulus in the Oaxaca focus, Mexico. Am J Trop Med Hyg 83(1): 21-27.

11. Higazi TB, Zarroug IM, Mohamed HA, Elmubarak WA, Deran TC, et al. (2013) Interruption of onchocerca volvulus transmission in the Abu Hamed focus, Sudan. Am J Trop Med Hyg 89(1): 51-57.

12. Tekle AH, Elhassan E, Isiyaku S, Amazigo UV, Bush S, et al. (2012) Impact of long-term treatment of onchocerciasis with ivermectin in Kaduna State, Nigeria: first evidence of the potential for elimination in the operational area of the African Programme for Onchocerciasis Control. Parasit Vectors 5: 28.

13. Hotez PJ (2007) Control of onchocerciasis-the next generation. Lancet. 369(9578): 1979-1980.

14. Albert Eisenbarth, David Ekale, Julia Hildebrandt, Mbunkah Daniel Achukwi, Adrian Streit, et al. (2013) Molecular evidence of Siisa form, a new genotype related to onchocerca onchengi in cattle from North Cameroon. Acta Trop 127(3): 261-265.

15. Post R J, Mustapha M, Kruger A (2007). Taxonomy and inventory of the cytospecies and cytopes of the simulium damnosum complex (Diptera:Smuliidae) in relation to Onchocerciasis. Trop Med Int Health 12(11): 1342-1353

16. Wahl G, Enyong P, Ngosso A, Schibel JM, Moyou R, et al. (1998) Onchocerca ochengi; epidemiological evidence of cross-protection against onchocerca volvulus in man. Parasitology 116(4): 349-362.

17. Higazi TB, Katholi CR, Mahmoud BM, Baraka OZ, Mukhtar MM, et al. (2001) Onchocerca volvulus: genetic diversity of parasite isolates from Sudan. Exp Parasitol 97(1): 24-34.

18. Lustigman S, McCarter JP (2007) Ivermectin Resistance in Onchocerca volvulus: Toward a Genetic Basis. PLoS Negl Trop Dis 1(1): 76.

19. Brieger WR, Awedoba AK, Eneanya CL, Hagan M, Ogbuagu KF, et al. (1998) The effects of ivermectin on onchocercal skin disease and severe itching: results of a multi-centre trial. Trop Med Int Health 3(12): 951961.

20. Cupp EW, Duke BO, Mackenzie CD, Guzman JR, Viera JC, et al. (2004) The effects of long-term community level treatment with ivermectin (Mectizan)on adult onchocerca volvulus in Latin America. Am J Trop Med Hyg 71(5): 602-607.

21. Duke BO, Zea Flores G J, Cupp EW, Munoz B (1991) Comparison of the effects of single dose and of four six-monthly doses of ivermectin on adult onchocerca volvulus. Am J Trop Med Hyg 45(1):132-137.

22. Diawara L, Traore MO, Badji A, Bissan Y, Doumbia K, et al. (2009) Feasibility of onchocerciasis elimination with ivermectin treatment in endemic foci in Africa: first evidence from studies in Mali and Senegal. PLoS Negl Trop Dis 21(3): 497.

23. Wegesa P (1970) The present status of onchocerciasis in Tanzania. A review of the distribution and prevalence of the disease. Trop Geogr Med 22(330: 345-351.

24. Mwaiko GL, Mtoi RS, Mkufya AR (1990) Onchocerciasis prevalence in Tanzania. Cen Afr J Med 36(4): 94-96. 
25. Makunde WH, FM Salum, JJ Massaga, MS Alilio (2000). Clinical and parasitological aspect of itching caused by onhocerciasis in Morogoro Tanzania. Ann Trop Med Parasitol 94(8): 793-799.

26. Brattig NW, Rathjens U, Ernst M, Geisinger F, Renz A, et al. (2000). Lipopolysaccharide-like molecules derived from Wolbachia endobacteria of the filarial onchocerca volvulus are candidate mediators in the sequence of inflammatory and antiinflamatory responses of human monocytes. Microbes Infect 2(10): 1147-1157.

27. Brattig NW, Bazzocchi C, Kirschning CJ, Reilling N, Buttner DW, et al (2004). The major surface protein of Wolbachia endosymbionts in filarial nematodes elicits immune responses through TLR2 and TLR4. Immunol 173(1): 437-445.

28. Tory P Johnson, Richa Tyagi, Paul R Lee, Myoung Hwa Lee, Kory R Johnson, et al. (2017) Nodding Syndrome may be an autoimmune reaction to the parasitic worm onchocerca volvulus. Sci Transl Med 9(377): 6953.

29. Kipp W, Burnham G, Bamuhiiga J, Leichsenring M (1996). The Nakalanga syndrome in Kabarole district, Western Uganda. Am J Trop Med Hyg 54(1): 80-83.

30. Christian Timmann, Esther van der Kamp, Andre Kleensang, Inke R Konig, Thorsten Thye, et al. (2008) Human genetic resistance to Onchocerca volvulus: evidence for linkage to chromosome $2 \mathrm{p}$ from an autosome-wide scan. J Infect Dis 198(3): 427-433.

31. Osei-Atweneboana MY, Eng JK, Boakye DA, Gyapong JO, Prichard RK (2007) Prevalence and intensity of Onchocerca volvulus infection and efficacy of ivermectin in endemic communities in Ghana: a two-phase epidemiological study. Lancet 369(9578): 2021-2029.

32. Mike Y Osei-Atweneboana, Kwablah Awadzi, Simon K Attah, Daniel A Boakye, John O Gyapong, et al (2011) Phenotypic evidence of emerging ivermectin resistance in Onchocerca volvulus. PLoS Negl Trop Dis 5(3): 998.

33. Raybould JN, White GB (1979). The distribution, bionomics and control of onchocerciasis vectors (Diptera: Simuliidae) in Eastern Africa and the Yemen. Tropenmed Parasitol 30(4): 505-547.

34. Maegga BT (1991) Onchocercaiasis : In Health and Disease in Tanzania (Mwaluko GMP, Kilama WL, Mandara MP, Murru M, and CNL Mcpherson, Editors; HarperCollins Academic London pp 177-188.

35. Maegga, BTA (1977) Summary of onchocerciasis epidemiological survey 1976/77, unpublished internal Report. Ministry of Health, Tanzania.

36. Anthony R Mawson, Williams H Makunde, Alan D Penman, Veronica de Los Angeles Hernandez Morales, Akili K Kalinga, et al (2017) Retinoid Expression in ochocercal Skin disease: Pilot study. Infect Dis 10: 1178633617731741

37. (TNOCP 1998) Tanzania National Onchocerciasis control Programme.

38. (TNOCP 2000) Tanzania National Onchocecriasis Control Programme.
39. Murdoch ME, Hay RJ, Mackenzie CD, Williams JF, Ghalib HW, et al. (1993) A clinical classification and grading system the cutaneous changes in onchocerciasis. Br J Dermatol 129(3): 260-269.

40. World Health organization (2001) Certification of Elimination of Human onchocercisis: Criteria and Procedures. Geneva: World Health Organization pp.1-36.

41. Mamadou O Traore, Moussa D Sarr, Alioune Badji, Yiriba Bissan, Lamine Diawara, et al. (2012) Proof-of principle of onchocerciasis elimination with ivermectin treatment in endemic foci in Africa: final results of a study in Mali and Senegal. PLoS Negl Trop Dis 6(9): 1825.

42. Afework HT, Elhassan E, Sunday I, Amazigo UV, Noma SM, et al. (2012) Impact of long-term treatment of onchocerciasis with ivermectin in Kaduna State, Nigeria: first evidence of the potential for elimination in the operational area of the African Programme for Onchocerciasis Control. Parasite Vector 5: 28.

43. Lamine Diawara, Mamadou O Traore, Alioune Badji, Yiriba Bissan, Konimba Doumbia, et al. (2009) Feasibility of onchocerciasis elimination with ivermectin treatment in endemic foci in Africa: first evidence from studies in Mali and Senegal. PLoS Negl Trop Dis, 3(7): 497.

44. Lakwo TL, Garms R, Rubaale T, Katabwara M, Walsh F, et al. (2013) The disappearance of Onchocerciasis from the Itwara focus, Western Uganda after elimination of the vector simulium neavei and 19 years of annual ivermectin treatments. Acta Trop 126(3): 218-221.

45. Katabwara M, Lakwo T, Habomugisha P, Aguyo S, Byamukana E, et al. (2014) Transmission of onchocerca volvulus by Simulium neavei in Mount Elgon focus of Eastern Uganda has been interrupted. Am J Trop Med Hyg 90(6): 1159-1166.

46. M T Rubio de Kromer 1, M Kromer, K Luersen, N W Brattig (1998) Detection of a chemotactic factor for neutrophils in extracts of female Onchocerca volvulus. Acta Trop 71(1): 45-56.

47. Amazigo UV (1993 Onchocerciasis and women's reproductive health; indigenous and biomedical concepts. Tropical Doctor 23(4): 149-151.

48. Amazigo UV (1994) Detrimental effects of onchocerciasis on marriage age and breast-feeding. Trop Geogr Med 46(5): 322-325.

49. C Vlassoff, M Weiss, E B Ovuga, C Eneanya, P T Nwel, et al. (2000) Gender and the stigma of onchocercal skin disease in Africa. Socl Sci Med 50(10):1353-1368

50. Nwoke BE (1990). The socio-economic aspects of human onchocerciasis in Africa: present appraisal. J Hyg Epidemiol Microbiol Immunol 34(1): 37-44.

51. Wagbatsoma VA, Okojie OH (2004) Psychosocial effects of river blindness in a rural community in Nigeria. J R Soc Promot Health 124: 134-136.

52. World Health Organization (1995) Onchocerciasis and its control. Report of a WHO Expert Committee on onchocerciasis control. Tech Rep Series 852: 1-104. 